\title{
High-throughput sequencing of forensic genetic samples using punches of FTA cards with buccal swabs
}

\author{
Marie-Louise Kampmann, Anders Buchard, Claus Børsting, and \\ Niels Morling \\ Section of Forensic Genetics, Department of Forensic Medicine, \\ Faculty of Health and Medical Sciences, University of Copenhagen, \\ Copenhagen, Denmark
}

${ }^{*}$ M.K. and A.B. contributed equally to this work.

BioTechniques 61:149-151 (September 2016) doi 10.2144/000114453

Keywords: massively parallel sequencing; FTA cards; DNA long-term storage; next-generation sequencing

Supplementary material for this article is available at www.BioTechniques.com/article/114453.

Here, we demonstrate that punches from buccal swab samples preserved on FTA cards can be used for high-throughput DNA sequencing, also known as massively parallel sequencing (MPS). We typed 44 reference samples with the HID-Ion AmpliSeq Identity Panel using washed $1.2 \mathrm{~mm}$ punches from FTA cards with buccal swabs and compared the results with those obtained with DNA extracted using the EZ1 DNA Investigator Kit. Concordant profiles were obtained for all samples. Our protocol includes simple punch, wash, and PCR steps, reducing cost and hands-on time in the laboratory. Furthermore, it facilitates automation of DNA sequencing.

High-throughput sequencing, or massively parallel sequencing (MPS), is being adapted for forensic investigation (1). The process has many steps, and any reduction in its complexity is welcome. Here, we describe how small punches of FTA cards (1.2 mm) containing buccal cells from mouth swabs can be used for high-throughput sequencing.

FTA cards are used for storage of biological samples such as blood and buccal swabs in forensic genetics laboratories. The DNA stored on the cards can be preserved for a long time at ambient temperatures. For this reason, FTA cards are frequently used for forensic genetics investigations (2-7).

The MPS kit HID-Ion AmpliSeq Identity Panel (Thermo Fisher Scientific, Waltham, MA) amplifies 124 single nucleotide polymorphisms (SNPs) in a single multiplex PCR (90 autosomal
SNPs and 34 Y-chromosome SNPs). A total of 44 reference buccal swab samples stored on Whatman FTA cards (GE Healthcare, Wauwatosa, WI) were typed using this kit and then sequenced on the Ion Torrent Personal Genome Machine (Ion PGM) (Thermo Fisher Scientific). The samples were typed in duplicate using two different input DNA sources in the initial PCR reaction. First, the DNA was extracted from FTA cards using the EZ1 DNA Investigator Kit (Qiagen, Hilden, Germany) and the BioRobot EZ1 Workstation (Qiagen), following the manufacturer's recommendations. The extracted DNA was quantified with a Qubit 3.0 Fluorometer using the Qubit dsDNA HS Assay Kit (Thermo Fisher Scientific) according to the manufacturer's instructions. One nanogram of purified DNA was used for AmpliSeq PCR amplification of the EZ1-extracted samples. Second, the samples were typed using $1.2 \mathrm{~mm}$ punches from FTA cards that were washed once with 200 $\mu \mathrm{L}$ molecular grade water (6). The wash was performed in microtiter plates. After adding water, the punches were shaken at $200 \mathrm{rpm}$ for $30 \mathrm{~min}$. The water was removed with a pipette, and the punches were left to dry for $1 \mathrm{~h}$ at room temperature. Subsequently, the PCR mix was applied. The conditions of the PCR, purification of amplicons, and quantification were identical for both methods for all subsequent steps. PCR was performed according to the manufacturer's (Thermo Fisher Scientific) instructions, with the exception that the number of PCR cycles was changed from 21 to 24 . Libraries were constructed by first adding $2 \mu \mathrm{L}$ FuPa reagent (Thermo Fisher Scientific) to the samples, barcoding was done using the Ion Express Barcode Adapters (Thermo Fisher Scientific), and purification was performed with AMPure XP Reagents (Agencourt, Beverly, MA). All reactions were performed according to the manufacturer's instructions. Libraries were quantified with a Qubit 2.0 Fluorometer using the Qubit dsDNA HS Assay Kit according to the

\section{METHOD SUMMARY}

Here, we demonstrate that small punches from buccal swab samples preserved on FTA cards can be used for high-throughput sequencing in forensic genetics. The method includes simple punch, wash, and PCR steps. 
A

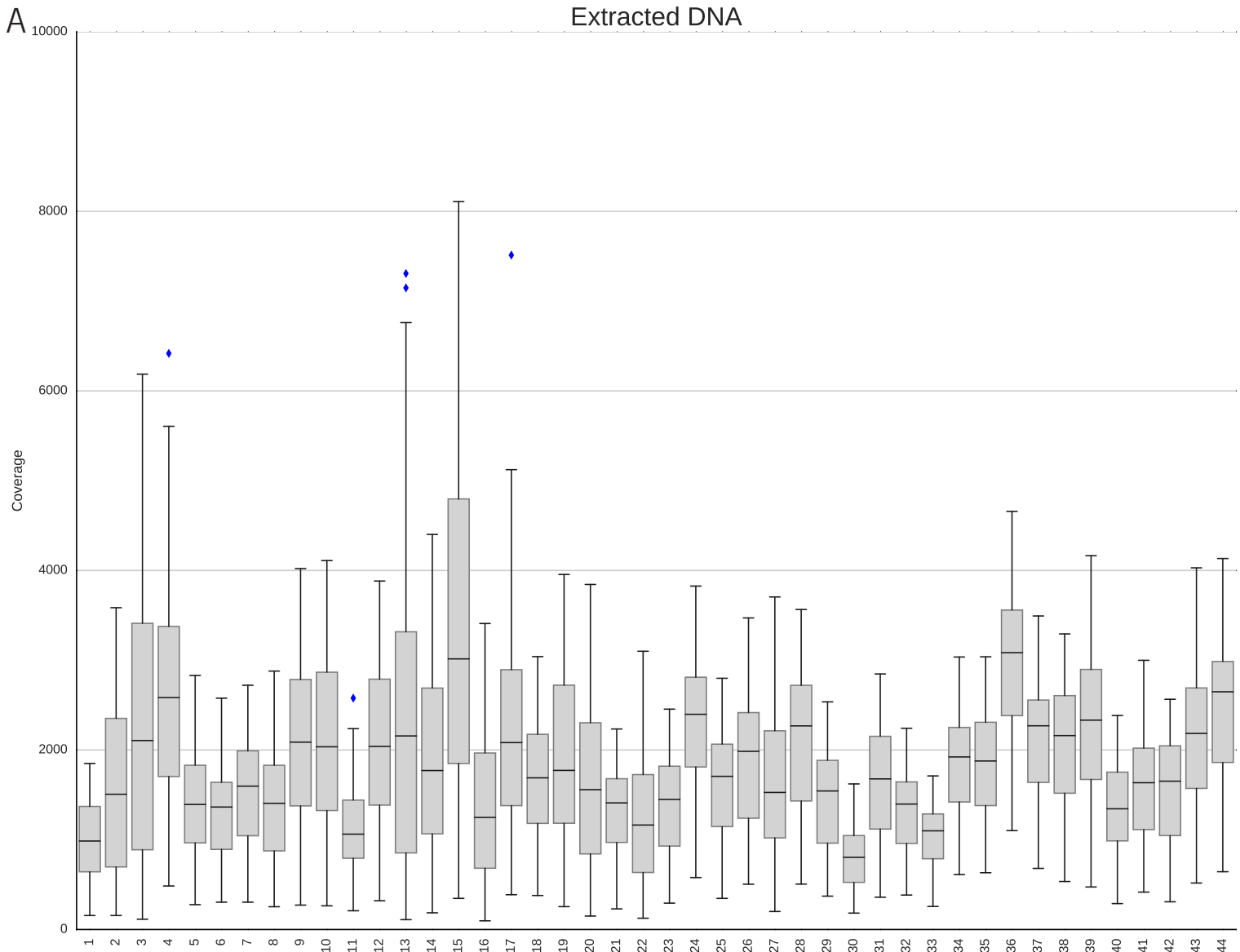

B

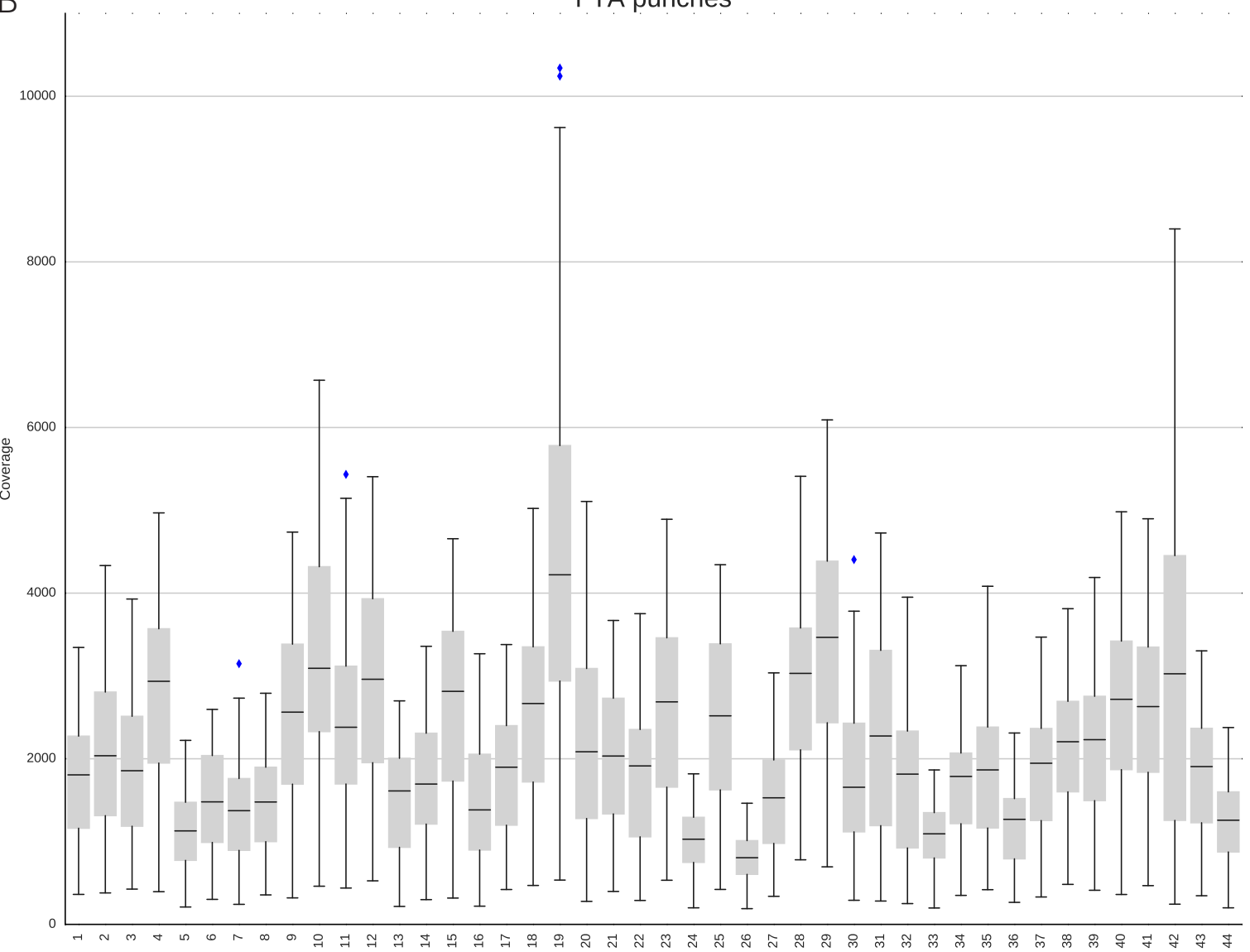

Figure 1. The median number of reads and the variation of read counts for all 124 SNPs in each sample.

(A) DNA amplified from EZ1-extracted samples. (B) DNA amplified from FTA card punches. Blue diamonds ( $\bullet$ represent outliers. 
manufacturer's instructions. Emulsion PCR and loading of the chips were done using the Ion Chef Instrument (Thermo Fisher Scientific), following the manufacturer's instructions. Two pools of 16 samples each were sequenced on two Ion 316 Chips v2, together with one positive and one negative control, using the Ion PGM IC 200 kit according to the manufacturer's (Thermo Fisher Scientific) instructions. The data were analyzed on a Torrent Server (version 4.2.1) using the HID_SNP_Genotyper Plugin version 4.2 (Thermo Fisher Scientific). The hotspot regions were defined in .bed files.

We aimed for a minimum read depth of at least 200 for all autosomal SNPs and a heterozygote balance $(\mathrm{Hb})>$ 0.3 . The profiles obtained with the two different starting materials were compared with each other. Complete concordance was obtained for all samples.

Figure $1, \mathrm{~A}$ and $\mathrm{B}$ shows the median numbers of reads and the variation of read counts for all 124 SNPs in the samples amplified from EZ1-extracted DNA and washed FTA card punches, respectively. In general, the average coverage was higher for the samples typed on FTA card punches than for those typed on EZ1 extracts $(P<0.01$, Wilcoxon test), and the sample-tosample variation of the coverage was larger for the FTA card punches than for EZ1-extracted DNA. The higher average coverage and larger sampleto-sample variation for the FTA card punches may reflect the variable amount of DNA on FTA card punches. The library DNA concentrations (mean $\pm \mathrm{SD}$ ) were $1.6 \pm 1.0$ and $1.9 \pm 1.4 \mathrm{ng} /$ $\mu l$ for the EZ1 purified samples and the punched FTA cards, respectively. The libraries were quantified and pooled in equal molar ratios prior to sequencing, which should reduce the difference in amplified DNA. However, in a sample with a low amount of input DNA in the initial PCR, the proportion of primerdimers compared with amplified DNA will be larger than that in a sample with a high amount of input DNA. Thus, the quantification of the purified libraries using the Qubit 2.0 Fluorometer may have been overestimated in samples with low amounts of input DNA in the initial PCR. The normalized inter-locus balances (Supplementary Table S1) and the heterozygous balances for each locus (Supplementary Table S2) were analyzed for the two different sources of input DNA. Student's $t$-test revealed that in some loci, the balances were significantly different. However, the differences were small and did not affect the genotype calls.

Our data showed that washed punches from FTA cards (filter papers) are well-suited for high-throughput DNA sequencing. The new method eliminates the need for extraction of DNA, saving money and hands-on time in the laboratory while also making the workflow easy to automate. It was recently shown by others (7) that FTA card punches could be used as sample material for sequencing 140 SNPs with a customized GeneRead (Qiagen) workflow designed for the MiSeq system (Illumina, San Diego, CA). The FTA card punches were washed three times with water in that protocol, whereas we used a single wash in this study.

\section{Author contributions}

M.K., A.B., C.B., and N.M. designed the study. M.K. and A.B. analyzed data and wrote the manuscript. C.B. and N.M. revised the manuscript

\section{Acknowledgments}

The authors thank Pernille Martini Jensen for the laboratory work, as well as Carina Grøntved Jønck and Brian Stidsen for help with the bioinformatics work.

\section{Competing interests}

The authors declare no competing interests.

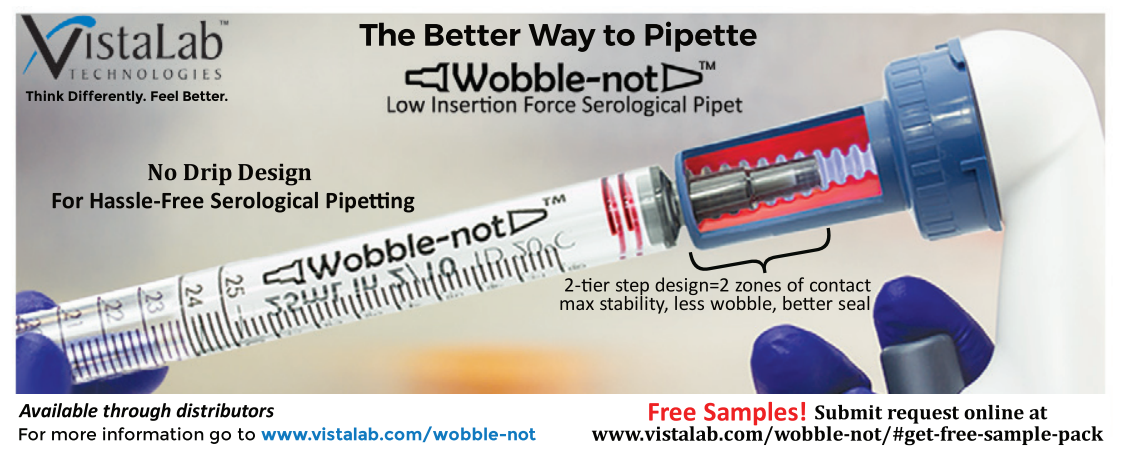

\section{References}

1. Børsting, C. and N. Morling. 2015. Next generation sequencing and its applications in forensic genetics. Forensic Sci. Int. Genet. 18:78-89.

2. Burgoyne, L., J. Kijas, P. Hallswort, and analysis of DNA from blood. Proceedings from the Fifth International Symposium on Human Identification.

3. Nagy, Z.T. 2010. A hands-on overview of tissue preservation methods for molecular genetic analyses. Org. Divers. Evol. 10:91105.

4. Johanson, H.C., V. Hyland, C. Wicking, and R.A. Sturm. 2009. DNA elution from buccal cells stored on Whatman FTA Classic Cards using a modified methanol fixation method. Biotechniques 46:309-311.

5. He, H., L. Argiro, H. Dessein, and C. Chevillard. 2007. Improved technique that allows the performance of large-scale SNP genotyping on DNA immobilized by FTAC technology. Infect. Genet. Evol. 7:128-132.

6. Børsting, C., J.J. Sanchez, and N. Morling. 2007. Forensic genetic DNA typing with PCR-based methods, p. 123-142. In S. Hughes and A. Moody (Eds.), PCR: Method Express. Scion Publishing Ltd., Bloxham, UK.

7. Grandell, I., R. Samara, and A. O. Tilmar. 2016. A SNP panel for identity and kindship testing using massive parallel sequencing. Int. J. Legal Med. 130:905-914

Received 19 February 2016; accepted 08 June 2016.

Address correspondence to Anders Buchard, Section of Forensic Genetics, Department of Forensic Medicine, Faculty of Health and Medical Sciences, University of Copenhagen, Frederik V's Vej 11, 2100 Copenhagen, Denmark. E-mail: Anders.Buchard@sund.ku.dk

To purchase reprints of this article, contact: biotechniques@fosterprinting.com J. Turner. 1994. Safe collection, storage and 